\title{
Secure Conference Key Distribution Schemes for Conspiracy Attack
}

\author{
Kicuji Lioyama \\ NTT Communication Scicnce Laboratories \\ Scika-cho, Soraliu-gun, Kyoto 619-02 Japan
}

\begin{abstract}
At the Eurocrypt'ss meeting, we proposed three identity-based conference key distribution schemes. At the Asiacrypt'91 meeting, Shimbo and Kawamura presented a conspiracy attacking method which worked against our schenes to disclose a user's secret information. This paper proposes an improved identity-based conference key distribution scheme to counter this altack.
\end{abstract}

\section{Introduction}

Since Diffie and Hellman proposed the public key distribution system (DH scheme), several advanced schemes and problems related to the DH scheme have been presented [ITT82, S85, 086, KOS7, Y87, KO88, MS8, LLH89, Y90, FM90, C190]. One direction which the advanced schemes have taken is to authenticate exchanged messages with each user's identification information. This is called an idenliby-based system. Another direction being taken is to generate a common key among two or more usets called a conference key. Several conference key distribution schemes have previously been presented [IT'T82. KOS8, LLHS9, CI90]. These schemes can be regarded as examples of general multiparty protocols [B91, MR91], in which each of $m$ members in a nelwork has a private inpul $x_{i}$. Together, the members would like to compute, correctly, privately and fairly, any computable function $F\left(x_{1}, \cdots, x_{m}\right)$. In particular, multiparty prolocols must be robust (secure) to guard against cheating members.

At the Eurocryph's $\$$ meeting, we proposed three identity-based conference key distribution schemes, constructed [or star, complete grapl and ring networks [KO88]. At the Asiacrypl'91 meeting, Shimbo and liawamura prescuted a method for attacking our schemes [or star and complete graph networks [SKOl]. 'They pointed out that a pivot user's secret information could be revealed by a conspiracy between two legal users' conspiracy by using the Euclidean algorithn. In order to counter their atlack, we propose improving the identity-based confcrence kcy distribution schemes by introducing new random variables.

\section{Improved Conference Key Distribution Schemes}

All identity-based conference key distribution schemcs consist of a center procedure and a user procedure as follows.

\section{[Center Procedure]}

A trusted center generates the following information:

- Three large primes $(p, q, r)$ and the partial product $N=p q$.

- Intcgers $(e, d)$ satislying the congruence:

$$
e d \equiv 1 \bmod L, \text { where } L=\operatorname{lcm}(p-1, q-1, r-1)
$$


- An integer $g$ which is a primitive clement over GF(p), GF( $q)$ and GF $(r)$.

- An integer $S_{i}$ which is derived from user $i$ 's idculification information $I_{i}$ as follows:

$$
S_{i}=I_{i}^{d} \bmod N r, \quad I_{i}=h\left(I D_{i}\right),
$$

where $I D_{i}$ is user $i$ 's original identifier, and $t$ is a public one-way hash function.

The above information is classified into three categories: a secret system key $(p, q, d)$, a public system key $(N, r, g, c)$, and a secret user kry $S_{i}$ for uscr $i$.

\section{[User Procedure]}

Let $m$ be the number of users in a group sharing a common conference key. For simplicity, a user procedure for a slar network is described here. One user becomes a "pivot user", who communicales with the other $(m-1)$ users belonging to the group. The procedures for interactions between the pivot user, user 1 , and one of the other users, user $j(2 \leq j \leq m)$ are summarized as follows.

Step 1: User j's procedure.

Step 1.1 Choose a randon number $P_{j}$ and compute its reciprocal $\bar{P}_{j}$ :

$$
P_{j} \bar{P}_{j} \equiv 1(\bmod (r-1))
$$

Step 1.2 Compute the following $\left(X_{j}, Y_{j}\right)$ :

$$
\begin{gathered}
X_{j}=g^{e P_{j}} \bmod N r \\
Y_{j}=S_{j} g^{h\left(X_{j} \|\left(T_{\text {ime }) P,}\right.\right.} \bmod N r .
\end{gathered}
$$

Step 1.3 Send $\left(I_{j}, X_{j}, Y_{j}\right.$, Timc $)$ to user 1.

Step 2: User 1's procedure.

Step 2.1 Check the time and whether the following congruence holds:

$$
Y_{j}^{e} / X_{j}^{h\left(X_{j} \| T i m e\right)} \equiv I_{j}(\bmod N r)
$$

If the congruence holds, user 1 is able to verify that the message is from user $j$. Step 2.2 Choose random numbers $R_{1}\left(0<R_{1}<r\right)$ and $Q_{1 j}\left(0<Q_{1 j}<N, 2 \leq j \leq m\right)$. Step 2.3 Compute the following $\left(A_{1 j}, B_{1 j}\right)$ :

$$
\begin{gathered}
A_{1 j}=\left(X_{j}+Q_{1^{2}{ }^{r}}\right)^{e R_{1}} \bmod N r \\
B_{1 j}=S_{1}\left(X_{j}+Q_{1 j} r\right)^{h\left(A_{1}, \| T^{\prime} i \operatorname{me}\right) R_{1}} \operatorname{nod} N r
\end{gathered}
$$

Step 2.4 Send $\left(I_{1}, A_{1 j}, B_{1 j}\right.$, Time) to user $j$.

Step 2.5 Compute the common key $K$ with

$$
K=g^{e^{2} R_{1}} \bmod r
$$

Step 3 User $j$ 's procedurc.

Step 3.1 Check the time and whether the following congruence holds: 


$$
B_{1 j}^{e} / A_{1 j}^{h\left(\lambda_{1}, \| \text { Iime }\right)} \equiv I_{1}(\bmod N r) .
$$

If the congruence holds, user $j$ is able to vorify that the message is from user 1. Step 3.2 Computc the common key $\stackrel{K}{ }$ with

$$
\begin{aligned}
K & =A_{1 j}^{\bar{P}} \bmod r \\
& =g^{e^{x_{1}} R_{j}} \bmod r .
\end{aligned}
$$

\section{Remarks:}

1. The term $\left(X_{j}+Q_{1 j} r\right)$ in variables $A_{1 j}$ and $B_{1 j}$ in this new version was represented by the torm $X_{j}$ in the previous version [KO88]. This improvement renders Shimbo and Kawamura's allack inefeclive. The details will be discussed in Section 4.

2. The exponents $h\left(X_{j} \|\right.$ Time $)$ and $h\left(A_{1 j}||\right.$ Time $)$ in this new version were previously represented by the exponents $X_{j}$ and $A_{1 j}$, respectively, where \| denotes concalenation. The usage of a time stanp with a public one-way lash function $h$ is effective in preventing a replay altack.

\section{Shimbo and Kawamura's Attacking Method}

Here, a brief description is given of Shimbo and Kawamura's altacking method [SK91] for the previous version where the term $X_{j}$ was used instead of the terms $\left(X_{j}+Q_{1 j} r\right)$ in the variables $A_{1 j}$ and $B_{1 j}$. Their attack requires a couspiracy between two users, other than user 1 , belonging to the group. The attacker's' aim is to disclose the pivot user's secret information $S_{1}$. Note that if this attack succeds, the attackers can pretend to be user 1 in the subsequent key generation procedurc. $\Lambda$ concrete altacking procedure is as follows. Assume that uscr 2 and user 3 conspire and user 2 becomes a "pivol conspirator". First, user 3 sends $\left(P_{3}, A_{13}, B_{13}\right)$ to user 2 . Next, user 2 computes $T$ :

$$
\begin{aligned}
T & =B_{12}^{P_{3} A_{13}} / B_{13}^{P_{2} A_{12}} \bmod N r \\
& =S_{1}^{P_{3} A_{13}-P_{2} A_{12}} \bmod N r .
\end{aligned}
$$

Since user 2 knows the valuc of the exponent $\left(P_{3} A_{13}-P_{2} A_{12}\right)$, denoted by $c$, and the relation:

$$
S_{1}^{e} \equiv I_{1} \bmod N r,
$$

the "Euclidean Attack" [SS3] can be applied as follows: if $e$ and $c$ are coprime, the integer solution $(x, y)$ satisfying $e x+c y=1$ can be easily obtained by the Euclidean algorithm. Then, $S_{1}$ is derived from $\left(I_{1}, T, x, y\right)$ with

$$
\begin{aligned}
I_{1}^{x r} T^{y} \bmod N r & =S_{1}^{r x+c y} \bmod N r \\
& =S_{1} \bmod N r .
\end{aligned}
$$

Finally, user 2 sends $S_{1}$ to user 3 . 
The probability of a successful autack being carried out can be estimated by the probability that $e$ and $c$ are coprime. If $P_{1}$ and $P_{2}$ are chosen as a coprime pair and $e=3$, then the probability of a successlul attack is about 0.67 .

It should be noted that this conspiracy atlacking method is vulnerable because the nonpivot conspirator (user 3 ) discloses his sccret information $P_{3}$. Once user 2 obtains the value of $P_{3}$, he can easily compute the valuc of $S_{3}$ with

$$
S_{3}=Y_{3} / g^{x_{3} P_{3}} \bmod N r
$$

Thus, the conspiracy altack is based on maintaining "trust" between the conspirators.

\section{Security of New Schemes}

The security of the improved sclicmes is based on the difficulty of deriving secret information from public kcys, transmilted messages and the other user's secrel keys. The secrecy of $(p, q, d)$ is based on the dilliculty of factoring a large number $N$, while the secrecy of $\left(P_{i}, \bar{P}_{i}, R_{i}, K, K^{\prime}\right)$ is based on the difficulty of computing a discrete logarithm over $G F(r)$. In the new version, the secrecy of $S_{i}$ is based on the difficulty of compuling $P_{i}$ or extracting the $e$-th roots mod $N$ when the factors of $N$ are unknown.

As pointed out in [Sk91], the previous version was attacked because only the fixed common random number $R_{1}$ was used to compute $A_{1 j}$ and $B_{1 j}(2 \leq j \leq m)$ for each user. As a result, $S_{1}^{Z} \bmod N r$ with a known integer $Z(\neq c)$ could easily be computed by canceling the random number $R_{1}$ through the conspiracy of two users.

An effective means of countering this allack is to introduce distinct random numbers $Q_{1 j}(2 \leq j \leq m)$ into old variables $\left(A_{1 j}, B_{i j}\right)$. It is clear that new variables $\left(A_{1 j}, B_{1 j}\right)$ salisfy the completeness properties which arc needed to authenticate user 1's identity and to generate a common conference key. Even if the conspirators compute the variables $T=$ $B_{12}^{P_{3} A_{20}} / B_{13}^{P_{2} A_{12}} \bmod N_{r}$ for the new version, $T$ cannot be expressed by $S_{1}^{Z} \bmod N r$ with a known integer $Z(\neq e)$.

Our proposed new schemes can be regarded as variants of the parallel version of the extended Fiat-Shamir scheme [FS86,FFS87,0088,GQS8]. Although the value of $\left(X_{j}+Q_{1 ;} r\right)$ $\bmod r$ is knowu by user $j$, the value of $\left(X_{1 j}+Q_{1 j} r\right) \bmod N$ is random and unknown to user $j(j \neq 1)$. Thus, the transmitted messages $B_{1 j}$ are independent of the secret $S_{1}$ and there are no additional information leaks about $S_{i}$ in our schemes. Formally speaking, the parallel version of the extended Fiat-Shamir is a non-transferable (weak zero-knowledge) interactive proof system [OO88,GQ88]. Thus, we have the following lemma.

Lemma (Non Transferability) In the new version of the identity-based conference key distribution schemes, no transfcrable information about a secret $S_{\mathrm{i}}$ is reveaded.

\section{Conclusion}

Improved interactive conference key distribution schemes were proposed to counter Shimbo and Kawamura's conspiracy attack. The introduction of new random variables was shown to be effective in preventing the disclosure of a user's secret hey in the interactive protocols. The new schemes require addilional time for the generation of $(m-1)$ random variables and $(m-1)$ additions modulo $N r$. The transmission efficiency of the new schemes is the same as that of the provious schemes. 


\section{Acknowledgements}

We wish to thank Kazuo Ohta (presently at MIT) and Michael Wang (University College, U.N.S.W.) for their valuable comments.

\section{References}

[B91] D. Beaver: "Foundations of Secure Interactive Computing", Proc. of C1RYPTO'91, pp.9-1-9-7 (1991).

[CI90] T. Chikazawa and T. Inoue: "A new key sharing system for global telecommunications", Proc. of GLOBCOM'90, pp.1069-1072 (1990).

[FM90] W. Fumy and M. Munzert: "A modular approach to key distribution", Proc. of CRYP'TO'90, pp.274-283 (1990).

[FS86] A. Fiat and A. Shamir: "How to prove yourself: Practical solutions to identification and signature problems", Proc. of CRYP'TO'86, pp.186-194 (1986).

[FF587] U. Feige, A. Fiat and A. Shamir: "Zcro knowledge prools of identity", Proc. of STOC, pp.210-217 (1987).

[GQ88] L.C. Guillou and J. J. Quisquarter: "A practical zero-knowledge protocol fitted to security microprocessor nuinizizing both transmission and memory", Proc. of Eurocrypt'88, pp.123$128(1990)$.

[ITT82] I. Ingemarson, D.T. Tang and C.K. Wong: “A conference key distribution system", IEEE Trans, on Information Theory, Vol. IT-28, pp.714-720, (1982).

[KO88] K. Koyama and K. Ohta: "Security of Improved Idenlity-based Conference Key Distribution Systems", Proc. of Eurocrypt'88, pp.11-19 (1989).

[LLH89] C.S. Lail, J.Y. Lee and L. Harn: "A new threshold scheme and its application in designing the conference key distribution cryplosystem", Information Processing Letters, Vol.32, No.3, pp.95-99 (1989).

[M88] K.S. McCurley: "A key distribution system equivalent to factoring", J. of Cryptology, Vol.1 , No. 2, pp.95-106, (1988).

[MR91] S. Micali and P. Rogaway: "Secure computation", Proc. of CRYPTO'91, p9-8 (1991).

[086] E. Okamoto: "Proposal for identity-based key distribution systems", Electronics Letters Vol.22 pp.1283. 1284 (1986).

[OO88] K. Ohta and T. Okamoto: "A modification of the l'iat-Shamir scheme", Proc. of CRYPTO'88, pp.232-243 (1988).

[S83] G. J. Simmons: "A "weak' privacy protocol using the RSA crypto algorithm", Cryptologia 7, 2, pp.180-182 (1983).

[S85] Z. Shmuely: "Composite Diflie-Hellman public-key generating systems are hard to break", TR. NO. 356, Computer Science Dept. Technion, IIT, Feb. (1985).

[SK91] A. Simbo and S. Kawanuma "Cryptanalysis of several conference key distribution sehenes", Proc. of Asiacrypt'91, pp.155-160 (1991).

[Y90] Y. Yacobi: "A key distribution "paradox", Proc. of CRYPTO'90, pp.268-273 (1990). 